\title{
Cirugía postbariátrica: el último eslabón en el protocolo de cirugía metabólica
}

\author{
Postbariatric surgery: the last chain-link in the metabolic surgery protocol \\ Dr. José Eduardo Telich-Tarriba,* Dr. Daniel Ángeles-Gaspar, \\ Dr. Lino Enrique Ramírez-Sosa, ${ }^{\S}$ Dr. José Telich-Vidal*
}

Palabras clave: Cirugía plástica, cirugía bariátrica, cirugía postbariátrica, contorno corporal, calidad de vida, pérdida masiva de peso.

Keywords: Plastic surgery, bariatric surgery, postbariatric surgery, body contouring, quality of life, massive weight loss.

\footnotetext{
* Departamento de Cirugía Plástica y Reconstructiva, Hospital Ángeles Pedregal. Programa de Postgrado en Cirugía Estética, Facultad Mexicana de Medicina Universidad La Salle. Ciudad de México, México.

¥ Unidad Médica de Alta Especialidad Hospital de Especialidades No. 1, Centro Médico Nacional del Bajío, Instituto Mexicano del Seguro Social. León, México.

$\S$ Unidad Médica de Alta Especialidad Hospital de Especialidades «Dr. Antonio Fraga Mouret», Centro Médico Nacional «La Raza», Instituto Mexicano del Seguro Social. México, Ciudad de México.
}

Recibido:

02 noviembre 2020 Aceptado:

23 noviembre 2020

\section{RESUMEN}

La cirugía bariátrica se ha convertido en un tratamiento efectivo y seguro para los casos severos de obesidad, sin embargo, a pesar del excelente resultado en cuanto a la pérdida ponderal, los pacientes que presentan pérdida masiva de peso por este tipo de procedimientos experimentan cambios morfológicos que alteran su imagen corporal, evitando que el resultado estético sea totalmente satisfactorio. Con el aumento en el número de cirugías bariátricas realizadas en los diversos centros hospitalarios, es importante que los cirujanos plásticos cuenten con herramientas que les permitan ofrecer tratamientos óptimos para mejorar los resultados postbariátricos, ya que esto impacta de manera positiva en la calidad de vida. Los principales objetivos de la cirugía plástica postbariátrica son eliminar los tejidos redundantes, mejorar el contorno corporal y así contrarrestar los impedimentos funcionales. Se presenta una revisión de la literatura atendiendo los principales aspectos de la cirugía plástica postbariátrica y las opciones terapéuticas de acuerdo con las principales áreas anatómicas afectadas.

\section{ABSTRACT}

Bariatric surgery has become an effective and safe treatment for severe cases of obesity; however, despite its excellent result in terms of weight loss, patients with massive weight loss due to this type of surgery develop morphological changes that alter their body image with shunning satisfactory post-surgical results. With the increase in the number of bariatric procedures performed in various hospitals, it is important that plastic surgeons have tools that allow them to offer optimal treatments to improve post bariatric results, impacting the quality of life of patients positively. The main goals of post bariatric plastic surgery are to remove redundant tissues, improve body contour, and decrease functional impairments. A review of the literature is presented, considering the main aspects of post bariatric plastic surgery and therapeutic options according to the main anatomical areas affected.

\section{INTRODUCCIÓN}

T a obesidad es una acumulación anormal ـ excesiva de grasa corporal que resulta dañina para la salud, ${ }^{1}$ definida por la Organización Mundial de la Salud como un índice de masa corporal (IMC) igual o superior a 30.0. ${ }^{2} \mathrm{La}$ obesidad y el sobrepeso son factores de riesgo importantes para el desarrollo de una gran cantidad de enfermedades crónico-degenerativas, entre las que destacan la diabetes, enferme- dades cardiovasculares y cáncer. ${ }^{3}$ En décadas recientes se ha observado un incremento dramático en la prevalencia de obesidad en el mundo. En México, el 36.3\% de los adolescentes y $72.5 \%$ de los adultos padecen sobrepeso y obesidad, ${ }^{4}$ lo que nos posiciona como uno de los países más obesos.

La cirugía bariátrica engloba un conjunto de procedimientos que tienen como objetivo la disminución del peso corporal y la remisión de las comorbilidades asociadas a la obesidad.

Citar como: Telich-Tarriba JE, Ángeles-Gaspar D, Ramírez-Sosa LE, Telich-Vidal J. Cirugía postbariátrica: el último eslabón en el protocolo de cirugía metabólica. Cir Plast. 2020; 30 (3): 172-179. https://dx.doi.org/10.35366/98343 
Está indicada en sujetos con obesidad mórbida (IMC > 40) u obesidad grado II (IMC entre 35 y 40), con comorbilidades asociadas..$^{5}$ Los procedimientos se clasifican de acuerdo con su mecanismo de acción en restrictivos y malabsortivos.

Los procedimientos restrictivos consisten en la reducción del volumen intragástrico, lo que produce una sensación de saciedad con ingestas pequeñas; algunos ejemplos son la gastroplastia vertical, la banda gástrica ajustable y balones intragástricos. ${ }^{6}$ Los procedimientos malabsortivos limitan la cantidad de nutrientes que pasan a la circulación portal al evitar que el alimento pase por un segmento intestinal, el bypass gástrico en $\mathrm{Y}$ de Roux es el procedimiento más realizado. ${ }^{6}$

La pérdida masiva de peso se define como una reducción ponderal superior al 50\% del peso original, que ocasiona alteraciones notorias de la imagen corporal. Prácticamente todas las regiones corporales desarrollan lipodistrofia y pliegues cutáneos redundantes, los que se pueden asociar a eritema intertriginoso, infecciones recurrentes, o limitación funcional, además de ser causa de estigmas psicológicos y sociales. ${ }^{7}$

La cirugía postbariátrica constituye el último eslabón en el protocolo de tratamiento del paciente con pérdida masiva de peso; sin embargo, únicamente el 10\% de ellos decide someterse a procedimientos de contorno corporal tras la cirugía bariátrica. ${ }^{8}$ Los principios de la cirugía de contorno corporal postbariátrica son similares en todas las regiones corporales: resecar el tejido redundante y mejorar la forma de la región afectada. ${ }^{9}$

\section{Presentación clínica}

Nueve de cada diez casos que solicitan cirugía postbariátrica son mujeres, principalmente en la cuarta década de la vida. Además de estética corporal, buscan resolver complicaciones asociadas con el exceso de piel, como lumbalgia, cervicalgia, limitación del movimiento, lesiones cutáneas por rozaduras, ulceraciones e infecciones intertriginosas. ${ }^{10}$ El estudio del paciente requiere un equipo multidisciplinario integrado por especialistas en nutrición, psicología, medicina interna, anestesiología, cirugía bariátrica y cirugía plástica. ${ }^{11}$
En la exploración física, se debe valorar la redundancia y elasticidad cutánea, distribución grasa, presencia de estrías, cicatrices y alteraciones en la integridad de la pared muscular, como diástasis de rectos o hernias. ${ }^{8,12}$ En mamas deberá realizarse una evaluación minuciosa, teniendo en cuenta el riesgo de cáncer de mama. ${ }^{13}$ Todo paciente debe tener un registro fotográfico adecuado que permita valorar los 360 grados de la anatomía del área a operar. ${ }^{14}$

Los individuos son candidatos a cirugía una vez que han alcanzado y mantenido su peso meta durante un periodo entre tres y seis meses tras la cirugía metabólica; el IMC deberá encontrarse idealmente entre 25 y 30.5,12

Consideraciones preoperatorias: se requiere una valoración médica integral a fin de controlar comorbilidades como diabetes, hipertensión arterial o cardiopatía isquémica. ${ }^{15}$ Más de un tercio de los pacientes que se someten a cirugía bariátrica requieren valoración psiquiátrica para conocer sus motivaciones y expectativas. ${ }^{16}$ Otro aspecto importante es la evaluación del estado nutricional, ya que la cirugía metabólica es causa de desarrollo de múltiples deficiencias nutricionales, entre las que destacan la anemia ferropénica, deficiencia de vitamina B12, calcio, zinc, vitaminas liposolubles (ADEK) y proteínas, por lo que se deben controlar antes de cualquier procedimiento. ${ }^{17}$

El tabaquismo debe suspenderse, ya que incrementa el riesgo de infección, dehiscencia de heridas, necrosis de los colgajos y tromboembolismo. ${ }^{18}$ Algunas intervenciones que reducen el riesgo de enfermedad tromboembólica son la deambulación temprana, el uso de prendas de compresión y la profilaxis con heparina de bajo peso molecular. ${ }^{19}$

El uso de profilaxis antibiótica disminuye significativamente el riesgo de infección postoperatoria. La recomendación actual incluye el uso de una cefalosporina de primera o segunda generación, o clindamicina/vancomicina en pacientes alérgicos, administradas dentro de los 60 minutos previos a realizar la primera incisión. ${ }^{20}$

Objetivos quirúrgicos: la cirugía busca contrarrestar los impedimentos funcionales, estéticos y psicológicos de la redundancia de la piel. ${ }^{21}$ Los procedimientos se agrupan de acuerdo con las regiones anatómicas de interés. 
La cirugía de contorno cervicofacial incluye la ritidectomía, platismoplastia y liposucción cervical. La cirugía de contorno corporal superior incluye los procedimientos mamarios (aumento, reducción, mastopexia), resección de ginecomastia, braquioplastia, torsoplastia y abdominoplastia reversa. Finalmente, la cirugía de contorno corporal inferior incluye los distintos tipos de abdominoplastia, musloplastia y pexia del monte de Venus. ${ }^{22}$

Siempre se deberán tomar en cuenta los deseos y necesidades de los individuos, sin embargo, la secuencia más común de procedimientos es iniciar con la abdominoplastia y procedimientos de contorno glúteo y cadera, seguidos por cirugía mamaria. ${ }^{23}$

Una herramienta preoperatoria útil es la escala de Pittsburgh, que evalúa la laxitud y redundancia cutánea de diez áreas anatómicas y otorga una calificación: 0 normal, 1 adiposidad excesiva, 2 adiposidad severa y 3 deformidades múltiples. Con este sistema, es posible objetivar mejor los hallazgos en la exploración física y trazar un plan quirúrgico. ${ }^{9,24}$

La decisión de combinar procedimientos deberá considerar el estado funcional del sujeto, sus comorbilidades, regiones anatómicas a tratar y la experiencia del equipo quirúrgico. ${ }^{23}$ Es recomendable evitar procedimientos con vectores de tensión opuestos, así como tiempos quirúrgicos prolongados, ya que aumentan el riesgo de complicaciones postoperatorias. ${ }^{25}$

\section{Características regionales y técnicas quirúrgicas}

Tronco y abdomen: el abdomen suele mostrar la mayor deformidad y es el motivo de consulta más frecuente. ${ }^{21}$ Como resultado de la pérdida de peso, se forma un delantal cutáneo anterior y ptosis del monte de venus, dando al tronco una apariencia de cono invertido ${ }^{26}$ (Figura 1). Algunos casos presentan adhesiones supraumbilicales, lo que puede limitar el movimiento del colgajo abdominal. ${ }^{27}$

Los objetivos en el manejo del abdomen es lograr un vientre plano, plicar la musculatura abdominal, reparar hernias y reposicionar el monte de venus. Además, el manejo circunferencial del abdomen tiene la capacidad de modificar el contorno de los muslos laterales y

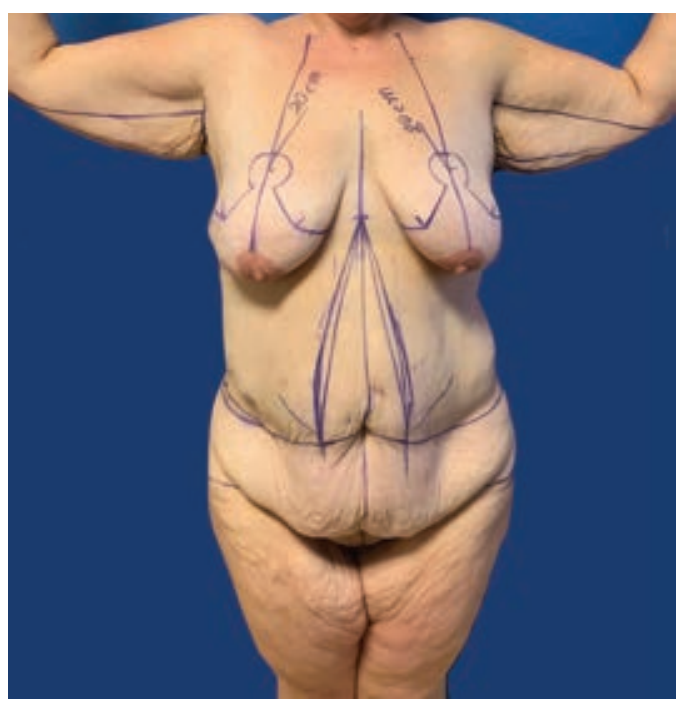

Figura 1: Se observa redundancia cutánea en brazos, región mamaria, abdomen, monte de venus y muslos tras la pérdida masiva de peso. Se aprecia marcaje preoperatorio para braquioplastia, mastopexia y abdominoplastia en flor de lis.

los glúteos, por lo que suele conocerse como un lower body lift. ${ }^{28}$

Las técnicas de abdominoplastia tradicionales no logran mejorar el contorno del tronco porque no tratan la laxitud del tejido lateral. ${ }^{28}$ La abdominoplastia en Flor de Lis o «T invertida» consiste en la resección del exceso cutáneo vertical y horizontal, logrando una reducción notable de la envoltura cutánea. ${ }^{29}$ La abdominoplastia circunferencial o en cinturón es un procedimiento capaz de manejar toda la circunferencia del tronco inferior, eliminando el panículo adiposo redundante, la ptosis del monte de Venus ayuda a definir la cintura, elevar los muslos y glúteos y eliminar el exceso de tejido posterior $^{30}$ (Figura 2). No hay un consenso para sugerir que una técnica de abdominoplastia se utilice sobre la otra, e incluso es posible combinar ambas técnicas en casos con gran redundancia cutánea. ${ }^{29,30}$

La paniculectomía se limita a resecar el excedente de piel y grasa de la pared abdominal en casos en los que el delantal cutáneo interfiere con las actividades cotidianas o genera problemas de higiene. Su principal diferencia con respecto a una abdominoplastia formal 
consiste en no tratar la laxitud muscular ni reposicionar la cicatriz umbilical. ${ }^{31}$

Glúteos: el objetivo quirúrgico es definir los glúteos, creando una línea de demarcación desde la parte posterior hasta los glúteos, alinear la cicatriz final que sigue la curva glútea superior en un patrón central de ala de gaviota, elevar los glúteos, incluido el pliegue central, cubrir el coxis con tejido blando adicional, desarrollar una curva ascendente del pliegue inferior del glúteo. ${ }^{32}$ Esto se logra con lipo-
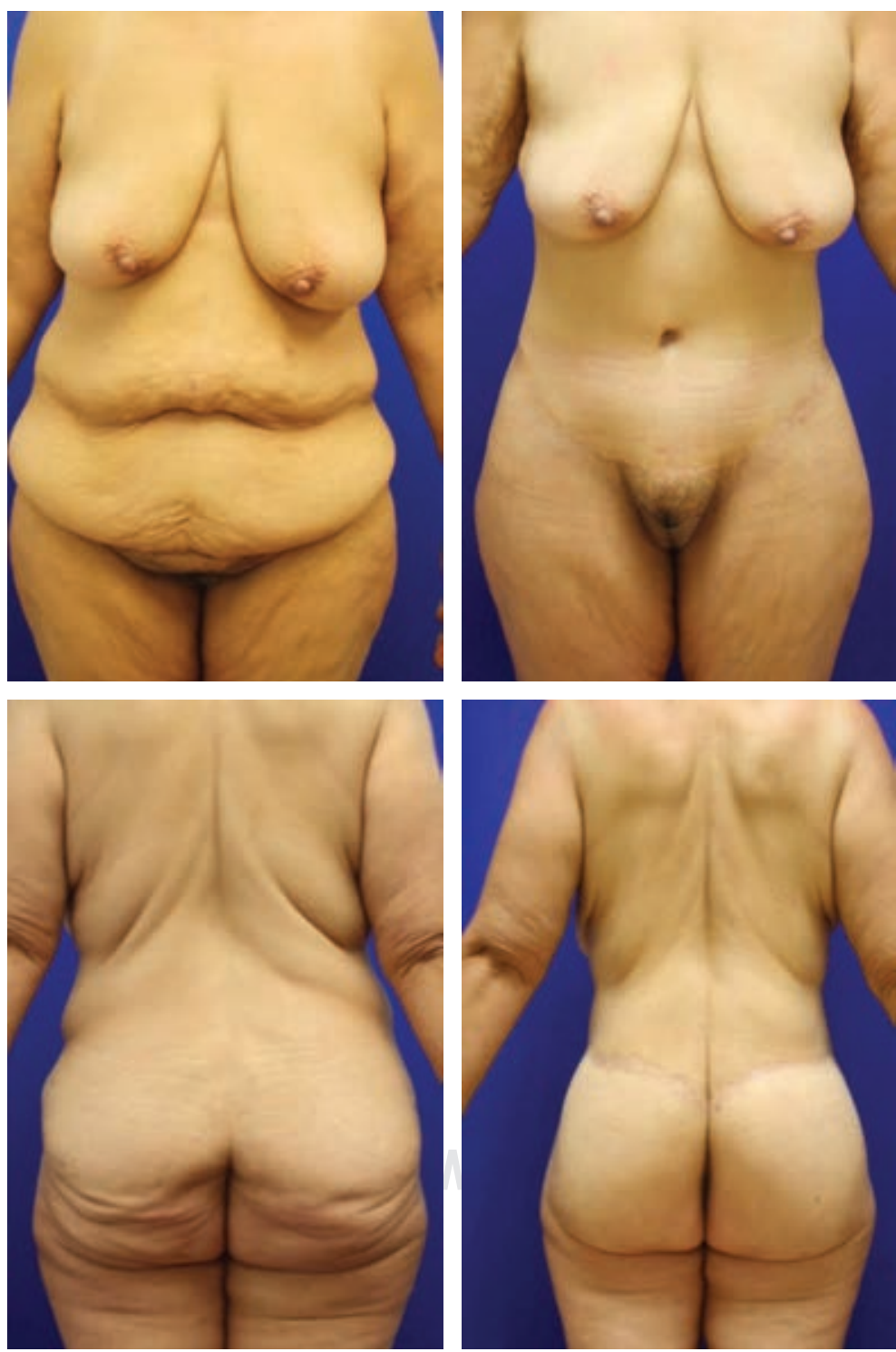

Figura 2: Se aprecia la corrección del contorno abdominal, glúteo y cadera después de la realización de una abdominoplastia circunferencial. succión y abdominoplastia circunferencial, también se debe considerar el aumento glúteo autógeno con injerto de grasa o colgajos dérmicos o adiposos para mejorar el contorno de los glúteos, especialmente para la proyección central. ${ }^{30,32}$

Muslos: el exceso de piel en los muslos es mayor en sentido horizontal que en vertical. El muslo debe dividirse conceptualmente en áreas laterales y mediales. La lipectomía circunferencial con elevación de la parte inferior del cuerpo es eficaz para definir la cintura y la porción lateral del muslo en un solo procedimiento. ${ }^{33}$ La musloplastia medial se debe realizar en un segundo procedimiento, ya que los vectores de tracción de la abdominoplastia y la musloplastia suelen ser opuestos. ${ }^{34} \mathrm{El}$ acceso vertical es más efectivo que el horizontal, se puede combinar con la liposucción de los muslos medial, anterior y posterior como adyuvante. ${ }^{35}$

El linfedema es una complicación catastrófica de la musloplastia medial, de tratamiento difícil y poco satisfactorio. El cirujano deberá ser extremadamente cuidadoso al momento de llevar a cabo la disección, a fin de no lesionar el paquete linfático localizado en el triángulo femoral. ${ }^{36}$

\section{Contorno corporal superior}

Cirugía mamaria: la cirugía de mama es compleja en este grupo de pacientes, puede haber pérdida de la forma, elasticidad, textura, sostén o volumen. Es importante conocer los deseos y expectativas de la paciente para determinar si se desea mantener, aumentar o reducir el volumen mamario. ${ }^{37}$

La mamoplastia de aumento se recomienda en casos sin ptosis, con volúmenes mamarios pequeños y adecuada calidad de la cubierta cutánea. Cuando se presente ptosis mamaria es recomendable realizar mastopexia ${ }^{38}$ (Figura 3). En algunos casos es posible combinar el procedimiento con la colocación de un implante mamario para aumentar el volumen o mejorar la proyección. ${ }^{39}$

La reducción mamaria está indicada en pacientes con macromastia. No existe una técnica estándar, por lo que el cirujano deberá adaptarse a las características del tejido de cada caso e individualizar el tratamiento. ${ }^{40}$ 

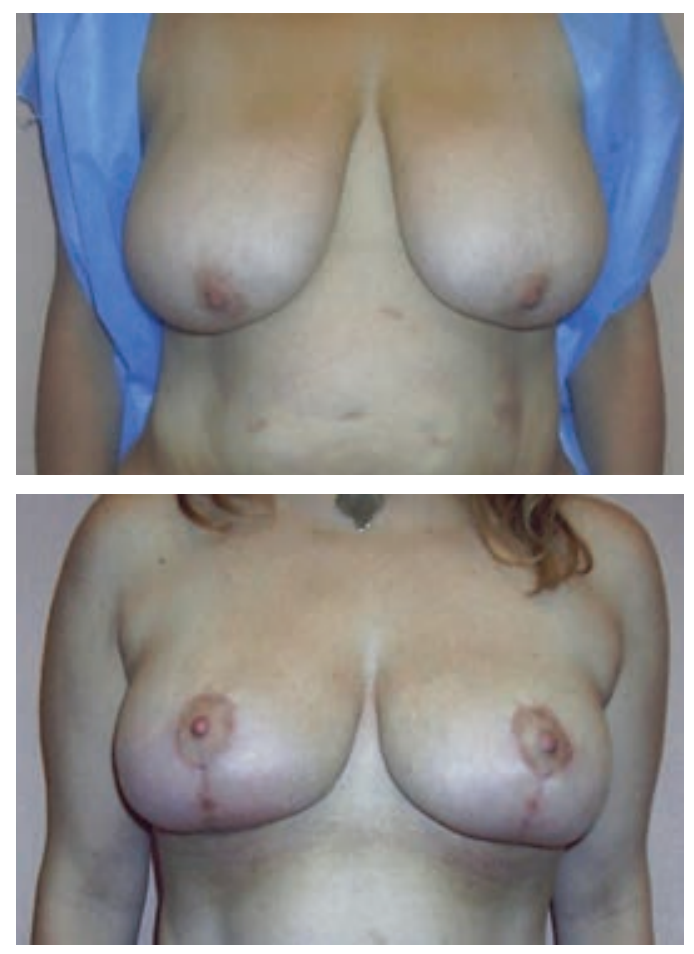

Figura 3: Se aprecia el estado pre- y postoperatorio en una paciente en la que se realizó mastopexia. La mama es una de las regiones con más variabilidad por la pérdida masiva de peso, por lo que se deberá evaluar en forma individual si la paciente requerirá únicamente pexia y aumento o reducción mamaria.

Ginecomastia: en los hombres, el manejo del tronco superior tiene como objetivo lograr un área pectoral plana, resecando el tejido mamario o prepectoral redundante y puede variar desde lipoaspiración y resecciones periareolares hasta la realización de mastectomía con cicatriz en T invertida. ${ }^{41}$

Brazos: los brazos pueden presentar redundancia cutánea severa desde el olécranon hasta la axila, e incluso extenderse hacia la región lateral del tórax. Las metas del tratamiento son eliminar el exceso de piel en brazo y tórax lateral, evitar bridas axilares y mejorar el contorno de la extremidad, limitando la extensión de las cicatrices. ${ }^{42}$

Con la braquioplastia se elimina el excedente cutáneo ocultando la cicatriz en el pliegue bicipital, en caso de extender la resección hacia el tórax es posible realizar una zetaplastia. ${ }^{42,43}$ Deberá evitarse un cierre a tensión, a fin de impedir el desarrollo de síndrome compartimental. ${ }^{43}$

Contorno cervicofacial: la pérdida de volumen gradual en la región facial y la redundancia de la piel ocasionan un aspecto de envejecimiento en los pacientes tras la pérdida masiva de peso. El objetivo quirúrgico es lograr una apariencia normal en la línea de la mandíbula y cuello por medio de la redistribución de piel en la región facial y cervical y armonizar la apariencia facial con el contorno del resto del cuerpo. ${ }^{44}$

Los procedimientos de cara y cuello son similares a los realizados con fines puramente estéticos, pero se debe tener en cuenta que en este grupo la ceja tiende a ser más ptótica y los pacientes tienden a presentar mayor redundancia en la región central del cuello. ${ }^{45}$

La ritidectomía tendrá como objetivo retirar el excedente cutáneo y dar un adecuado manejo al sistema musculoaponeurótico. ${ }^{46}$ En algunos casos es recomendable aplicar lipoinjertos con el fin de mejorar el volumen y armonía facial. ${ }^{47}$

Lipoaspiración: la lipoaspiración o liposucción por sí sola es incapaz de manejar adecuadamente el contorno corporal del paciente postbariátrico; sin embargo, es un complemento útil en todas las regiones corporales, permitiendo obtener resultados armónicos y en ocasiones facilitar la disección de los tejidos. ${ }^{48}$

\section{Cuidados postoperatorios}

El manejo postoperatorio de los pacientes deberá estandarizase. Se recomienda seguir todas las medidas de tromboprofilaxis postoperatoria. ${ }^{49}$

Se sugiere colocar drenajes para vigilar y prevenir la formación de seromas. ${ }^{50}$ Algunos autores recomiendan aplicar bloqueos nerviosos intercostales y pararrectales para reducir el tiempo de recuperación y la necesidad de analgésicos. ${ }^{51}$

El uso de prendas de compresión pretende reducir el riesgo de seroma, evitando las fuerzas de cizallamiento, ayudando a disminuir el espacio muerto; se recomienda su uso por lo menos por un mes. ${ }^{52}$

\section{Complicaciones}

Las complicaciones posteriores a la cirugía del contorno corporal postbariátrica son comunes, 
debido a la presencia de comorbilidades y dependen en gran medida del índice de masa corporal inicial. ${ }^{53}$

Inmediatas: pueden ser fatales y pueden incluir trombosis venosa profunda, embolia pulmonar, embolia grasa y hematomas. ${ }^{54} \mathrm{La}$ incidencia de trombosis venosa profunda es del $1.1 \%$ y de embolia pulmonar del $0.8 \% .{ }^{55}$

Tempranas: incluyen infección, dehiscencia de heridas, necrosis cutánea, seroma y edema prolongado. La dehiscencia puede ocurrir hasta en el 30\% de los casos y suele relacionarse con la extensión de las heridas y la tensión del cierre. ${ }^{56}$ La necrosis cutánea ocurre en el $10 \%$ de los casos, es resultado de la reducción en el flujo sanguíneo por tensión excesiva o adelgazamiento de los colgajos. ${ }^{57}$

Los linfoceles requieren de drenaje percutáneo con colocación de succión cerrada. En caso de fallar este manejo, se procederá a la exploración quirúrgica con ligadura de vasos linfáticos con fugas. ${ }^{34,36}$

El síndrome compartimental es una complicación grave en cirugía de contorno de extremidades. Deberá evitarse realizando cierres cutáneos sin tensión. En caso de presentarse, se deben liberar los vendajes de la extremidad $y$, si es necesario, liberar suturas de la herida. ${ }^{58}$

Tardías: incluyen asimetrías en el contorno, recurrencia de la diastasis del recto abdominal, cicatrices de mala calidad, o conos de rotación. Suelen correlacionarse con deficiencias en la planificación preoperatoria. ${ }^{53}$

\section{CONCLUSIONES}

La cirugía de contorno corporal posterior a pérdida masiva de peso tiene un impacto positivo en la calidad de vida de los pacientes. Con el aumento en la prevalencia de obesidad en el ámbito mundial, los procedimientos bariátricos se realizan cada vez en más centros quirúrgicos y derivado del éxito creciente tras su realización, los cirujanos plásticos deben estar familiarizados con el manejo perioperatorio y las diversas técnicas quirúrgicas disponibles para contribuir en la mejoría del aspecto físico en estos casos. La integración de clínicas multidisciplinarias es vital para brindar una atención especial y lograr cirugías seguras con resultados satisfactorios.

\section{REFERENCIAS}

1. Rodrigo S, Soriano JM, Merino JF. Causas y tratamiento de la obesidad. Nutr Clin Diet Hosp 2017; 37 (4): 8792.

2. Bray GA, Heisel WE, Afshin A et al. The science of obesity management: An endocrine society scientific statement. Endocr Rev 2018; 39 (2):79-132.

3. Fastenau J, Kolotkin RL, Fujioka K, Alba M, Canovatchel W, Traina S. A call to action to inform patient-centred approaches to obesity management: Development of a disease-illness model. Clin Obes 2019; 9 (3): 1-10.

4. Díaz RG, Candia MC, Martinez TJ, Esparza J. Lifestyle intervention for obesity: a call to transform the clinical care delivery system in Mexico. Diabetes Metab Syndr Obes 2019; 12: 1841-1859.

5. Wolfe BM, Kvach E, Eckel RH. Treatment of obesity: Weight loss and bariatric surgery. Circ Res 2016; 118 (11): 1844-1855.

6. Di Lorenzo N, Antoniou SA, Batterham RL et al. Clinical practice guidelines of the European Association for Endoscopic Surgery (EAES) on bariatric surgery: update 2020 endorsed by IFSO-EC, EASO and ESPCOP. Surg Endosc 2020; 34: 2332-2358.

7. Busetto L, Dicker D, Azran C et al. Practical recommendations of the obesity management task force of the European Association for the Study of Obesity for the post-bariatric surgery medical management. Obes Facts 2017; 10: 597-632.

8. Gunnarson GL, Frøyen JK, Sandbu R, Thomsen JB, Hjelmesæth J. Plastic surgery after bariatric surgery. Tidsskr Nor Laegeforen 2015; 135 (11): 1044-1049.

9. Song AY, Jean RD, Hurwitz DJ, Fernstrom MH, Scott JA, Rubin JP. A classification of contour deformities after bariatric weight loss: the Pittsburgh Rating Scale. Plast Reconstr Surg 2005; 116 (5): 1535-1544.

10. Cabbabe S. Plastic surgery after massive weight loss. Mo Med 2016; 113 (3): 202-206.

11. Neff KJ, Olbers T, le Roux CW. Bariatric surgery: the challenges with candidate selection, individualizing treatment and clinical outcomes. BMC Med 2013; 11 (8): 1-17.

12. Singh D, Forte AJ, Zahiri HR et al. Prognostication for body contouring surgery after bariatric surgery. Eplasty 2012; 12: 396-402.

13. Jager P, Wolicki A, Spohnholz J, Senkal M. Sex-specific aspects in the bariatric treatment of severely obese women. Int J Environ Res Public Health 2020; 17: 1-27.

14. Ivezaj V, Grilo CM. The complexity of body image following bariatric surgery: A systematic review of the literature. Obes Rev 2018; 19 (8): 1116-1140.

15. Schauer DP, Arterburn DE, Wise R et al. Predictors of bariatric surgery among an interested population. Surg Obes Relat Dis 2014; 10 (3): 547-522.

16. Yen YC, Huang CK, Tai CM. Psychiatric aspects of bariatric surgery. Curr Opin Psychiatry 2014; 27 (5): 374-379.

17. Mechanick JI, Youdim A, Jones DB et al. Clinical practice guidelines for the perioperative nutritional, metabolic and nonsurgical support of the bariatric surgery patient-2013 update: Cosponsored by American Society for Metabolic and Bariatric surgery. Obesity 2013; 21 (1): 1-64. 
18. Elrazek AE, Elbanna AE, Bilasy SE. Medical management of patients after bariatric surgery: principles and guidelines. World J Gastrointest Surg 2014; 6 (11): 220-228.

19. Barlett MA, Mauck KF, Daniels PR. Prevention of venous thromboembolism in patients undergoing bariatric surgery. Vasc Health Risk Manag 2015; 11: 461-477.

20. Chopra T, Zhao JJ, Alangaden G, Wood MH, Kaye KS. Preventing surgical site infections after bariatric surgery: value of perioperative antibiotic regimens. Expert Rev Pharmacoecon Outcomes Res 2010; 10 (3): 317-328.

21. Zwaan M, Georgiadou E, Stroh C et al. Body image and quality of life in patients with and without body contouring surgery following bariatric surgery: a comparison of pre- and post-surgery groups. Front Psychol 2014; 5: 1-10.

22. Paul MA, Opyrchal J, Knaklewicz M et al. The long-term effect of body contouring procedures on the quality of life in morbidly obese patients after bariatric surgery. PLOS ONE 2020; 15 (2): 1-10.

23. Song $P$, Patel NB, Gunther $S$ et al. Body image and quality of life: changes with gastric bypass and body contouring. Ann Plast Surg 2016; 76 (3): 216-221.

24. Zammerilla LL, Zou RH, Dong ZM et al. Classifying severity of abdominal contour deformities after weight loss to aid in patient counseling: a review of 1006 cases. Plast Reconstr Surg 2014; 134 (6): 888-894.

25. Beek ES, Molen AM, Ramshorst B. Complications after body contouring surgery in post-bariatric patients: The importance of a stable weight close to normal. Obesity Facts 2011; 4: 61-66.

26. Swedenhammar E, Stark B, Hallstrand AH, Ehrstom, Gahm J. Surgical training and standardized management guidelines improved the 30-day complication rate after abdominoplasty for massive weight loss. World J Surg 2018; 42: 1647-1654.

27. Karip B, Altun H, Iscan Y et al. Difficulties of bariatric surgery after abdominoplasty. Case Rep Surg 2014; 620175. doi: 10.1155/2014/620175.

28. Jacobs J, Schechner S, Jacobs JS. Abdominoplasty following massive weight loss. Semin Plast Surg 2006; 20 (1): 15-23.

29. Yazar SK, Serin M. Comparison of aesthetic quality of the final scar in abdominoplasty with conventional and mini inverted t-scar. Medicine 2019; 55: 1-8.

30. Ballestas-Campo O, Pomerane A. Abdominoplastia y pexia de pubis: estandarización de medidas. Cir Plast Iberolatinoam 2014; 40 (2): 149-158.

31. Esteban JR, Simón E, Delgado T, García JM, Llinás A. Paniculectomía masiva en lipodistrofia abdominal gigante: cuando el beneficio supera los riesgos. Rev Hispanoam Hernia 2016; 4 (4): 173-178.

32. Gerut ZE. Post-bariatric buttock contouring with autogenous tissue augmentation. Semin Plast Surg 2006; 20 (1): 30-37.

33. Shirvastava P, Aggarwal A, Khazanchi RK. Body contouring surgery in a massive weight loss patient: an overview. Indian J Plast Surg 2008; 41: 114-129.

34. Labardi L, Pietro G, Giglotti S et al. Medial Thighplasty: Horizontal and vertical procedures after massive weight loss. J Cutan Aesthet Surg 2012; 5 (1): 20-25.
35. Carney MJ, Matatov T, Freeman M et al. Clinical, biomechanical and anatomic investigation of Colles fascia and pubic ramus periosteum for use during medial thighplasty. Ann Plast Surg 2018; 80: 30-31.

36. Sisti A, Cuomo R, Zerini I et al. Complications associated with medial thigh lift: a comprehensive literature review. J Cutan Aesthet Surg 2015; 8: 191-197.

37. Hurwitz DJ, Golla D. Breast reshaping after massive weight loss. Semin Plast Surg 2004; 18 (3): 179-187.

38. Colicchia G, Di Pietro V, Cervelli V. Mastoplasty after massive weight loss: Redefinition and stabilization of the breast mound with submuscular autoprosthesis. J Cutan Aesthet Surg 2019; 12 (3): 164-173.

39. Bertozzi N, Pesce M, Santi P, Raposio E. One-stage immediate breast reconstruction: A concise review. Biomed Res Int 2017; 2017: 1-12.

40. Ngaage LM, Cantab MA, BChir MB et al. A12-year review of patient-reported outcomes after reduction mammoplasty in patients with high body mass index. Medicine 2019; 98 (25): 1-5.

41. Brown RH, Chang DK, Siy R, Friedman J. Trends in the surgical correction of gynecomastia. Semin Plast Surg 2015; 29 (2): 122-129.

42. Di Pietro V, Colicchia GM, Cervelli v, Gentile P. Arm contouring after massive weight loss: Liposuctionassisted brachioplasty versus standard technique. J Cutan Aesthet Surg 2018; 11 (2): 73-78.

43. Sisti A, Cuomo R, Milonia L et al. Complications associated with brachioplasty: a literature review. Acta Biomed 2017; 88 (4): 393-402.

44. Waltzman J, Zins J, Couto R. Face and neck lifting after weight loss. Clin Plast Surg 2019; 46 (1): 105-114.

45. Anlatici R, Ozerdem G, Demiralay S, Ozerdem OR. Face-neck lifting and ancillary procedures. A series of 203 cases. Medicine 2018; 97 (39): 1-8.

46. Hamra ST. Building the composite face lift: A personal odyssey. Plast Reconstr Surg 2016; 138 (1): 85-96.

47. Ma L, Wen H, Jian X et al. Cell-assisted lipotransfer in the clinical treatment of facial soft tissue deformity. Plast Surg 2015; 23 (3): 199-202.

48. Langer V, Singh A, Aly AS, Cram AE. Body contouring following massive weight loss. Indian J Plast Surg 2011; 44 (1): 14-20.

49. Almarshad FM, Almegren M, Alshuaibi $T$ et al. Thromboprophylaxis after bariatric surgery. Blood Res 2020; 55 (1): 44-48.

50. Reiffel AJ, Berie PS, Spector JA. A multi-disciplinary review of the potential association between closedsuction drains and surgical site infection. Surg Infect 2013; 14 (3): 244-269.

51. Lange M, Lee C, Knisely T et al. Efficacy of intravenous acetaminophen in length of stay and postoperative pain control in laparoscopic Roux-en-Y gastric bypass surgery patients. Bariatr Surg Pract P 2018; 13 (3): 103-108.

52. Aho JM, Nickerson TP, Thiels CA, Saint-Cyr M, Farley DR. Prevention of postoperative seromas with dead space obliteration. Int J Surg 2016; 29: 70-73.

53. Beidas O, Gusenoff J. Common complications and management after massive weight loss patient safety in plastic surgery. Clin Plast Surg 2018; 46 (1): 115122. 
54. Froehling DA, Daniels PR, Mauck KF et al. Incidence of venous thromboembolism after bariatric surgery: a population-based cohort study. Obes Surg 2013; 23 (11): 1874-1879.

55. Griffin M, Akhavani MA, Muirhead N et al. Risk of thromboembolism following body-contouring surgery after massive weight loss. Eplasty 2015; 15: 145-154.

56. Romano L, Zoccali G, Orsini G, Giuliani M. Reducing complications in post-bariatric plastic surgery: our experience and literature review. Acta Biomed 2019; 90 (4): 475-481.

57. Poodt IG, Dijk MM, Klein S, Hoogbergen MM. Complications of lower body lift surgery in postbariatric patients. Plast Reconstr Surg Glob Open 2016; 4 (9): 1-7.
58. Han HH, Lee MC, Kim SH et al. Upper arm contouring with brachioplasty after massive weight loss. Arch Plast Surg 2014; 41 (3): 271-276.

\section{Correspondencia:}

\section{Dr. José Eduardo Telich Tarriba}

Camino a Santa Teresa Núm. 1055-1180,

Col. Héroes de Padierna, 10700,

Alcaldía La Magdalena Contreras,

Ciudad de México, CDMX.

E-mail: josetelich@gmail.com

Conflicto de intereses: Los autores declaran no tener conflicto de intereses. 